\title{
A Topologically Charged Rotating Black Hole in the Brane
}

\author{
Alexis Larrañaga, ${ }^{1}$ Claudia Grisales, ${ }^{2}$ and Manuel Londoño ${ }^{2}$ \\ ${ }^{1}$ National Astronomical Observatory, National University of Colombia, Bogotá 11001000, Colombia \\ ${ }^{2}$ Department of Physics, National University of Colombia, Bogotá 11001000, Colombia
}

Correspondence should be addressed to Alexis Larrañaga; ealarranaga@unal.edu.co

Received 12 June 2013; Accepted 21 August 2013

Academic Editor: Kingman Cheung

Copyright (C) 2013 Alexis Larrañaga et al. This is an open access article distributed under the Creative Commons Attribution License, which permits unrestricted use, distribution, and reproduction in any medium, provided the original work is properly cited.

\begin{abstract}
We have obtained a rotating black hole solution in the braneworld scenario by applying the Newman-Janis algorithm. The new solution carries two types of charge, one arising from the bulk Weyl tensor and one from the gauge field trapped on the brane. In order to obtain this result, we used a modified version of the algorithm in which the involved complexification is the key point. The analysis of the horizon structure of the new metric shows similarities to the Kerr-Newman solution. In particular, there is a minimal mass to which the black hole can decay through the Hawking radiation. From the thermodynamical analysis, the possibility of a degenerate horizon gives a temperature that, instead of a divergent behaviour at short scales, admits both a minimum and a maximum before cooling down towards a zero temperature remnant configuration.
\end{abstract}

\section{Introduction}

There is a growing interest in the braneworld scenario which describes our universe as a brane embedded in a higher dimensional bulk. The brane supports all gauge fields excluding the gravitational field that lives in the whole spacetime. One of the most interesting scenarios is Randall-Sundrum II model in which a single brane lives in a $\mathbb{Z}_{2}$-symmetric, 5-dimensional, asymptotically anti-de-Sitter bulk [1], and black hole solutions on this model are particularly interesting because they have considerably richer physical aspects than black holes in general relativity [2-9].

As shown by Whisker [10], the generation of rotating solutions on the brane is not an easy task. In this work, we use the Newman-Janis algorithm $[11,12]$ which is regarded as a short cut to obtain spinning black hole solutions from the corresponding nonrotating ones. Up to the present, there is no reason as to why this method should work. Indeed, there is no full, clear explanation of why the method works and exactly what conditions must be satisfied in order for it to be successful in generating a rotating solution from a static, spherically symmetric seed solution. Drake and Szekeres [13] claim that the algorithm works only for vacuum solutions or in the case of a Maxwell energy-momentum tensor. However, the algorithm has been extended to be used in other scenarios (see, e.g., $[10,14,15]$ ).

In order to obtain the correct solution, we apply the modified prescription of the Newman-Janis algorithm presented by Modesto and Nicolini [14] to the particular solution in the braneworld model obtained by Chamblin et al. $[16,17]$ which carries two types of charge, one arising from the bulk Weyl tensor and one from the gauge field trapped on the brane. The result is a rotating black hole metric on the brane with the two charges and that resembles Kerr-Newmann solution. Finally, we also present the horizons structure and the temperature associated with the rotating braneworld black hole.

\section{The Braneworld Model}

The gravitational field on the brane is described by the Gauss and Codazzi equations of 5-dimensional gravity [18],

$$
G_{\mu \nu}=-\Lambda g_{\mu \nu}+8 \pi G T_{\mu \nu}+\kappa_{5}^{4} \Pi_{\mu \nu}-E_{\mu \nu}
$$

where $G_{\mu \nu}=R_{\mu \nu}-(1 / 2) g_{\mu \nu} R$ is the 4-dimensional Einstein tensor, $\kappa_{5}$ is the 5 -dimensional gravity coupling constant,

$$
\kappa_{5}^{4}=\left(8 \pi G_{5}\right)^{2},
$$


with $G_{5}$ being the gravitational constant in five dimensions, and $\Lambda$ is the 4-dimensional cosmological constant that is given in terms of the 5-dimensional cosmological constant $\Lambda_{5}$ and the brane tension $\tau$ by

$$
\Lambda=\frac{\kappa_{5}^{2}}{2}\left(\Lambda_{5}+\frac{\kappa_{5}^{2}}{6} \tau^{2}\right) .
$$

$T_{\mu \nu}$ is the stress-energy tensor of matter confined on the brane, $\Pi_{\mu \nu}$ is a quadratic tensor in the stress-energy tensor given by

$$
\Pi_{\mu \nu}=\frac{1}{12} T T_{\mu \nu}-\frac{1}{4} T_{\mu \sigma} T_{\nu}^{\sigma}+\frac{1}{8} g_{\mu \nu}\left(T_{\alpha \beta} T^{\alpha \beta}-\frac{1}{3} T^{2}\right)
$$

with $T=T_{\sigma}^{\sigma}$, and $E_{\mu \nu}$ is the projection of the 5-dimensional bulk Weyl tensor $C_{A B C D}$ on the brane $\left(E_{\mu \nu}=\delta_{\mu}^{A} \delta_{\nu}^{B} C_{A B C D}\right.$ $n^{A} n^{B}$ with $n^{A}$ being the unit normal to the brane). $E_{\mu \nu}$ encompasses the nonlocal bulk effect and its only known property is that it is traceless; that is, $E_{\sigma}^{\sigma}=0$.

A black hole type solution of the field equation (1) with $\Lambda=0$ and assuming that the localised gauge field on the brane is the Maxwell field is given by the line element

$$
d s^{2}=f(r) d t^{2}-\frac{d r^{2}}{f(r)}-r^{2} d \Omega^{2},
$$

where $d \Omega^{2}=d \theta^{2}+\sin ^{2} \theta d \varphi^{2}$ is the line element of the twosphere $S^{2}$ and

$$
f(r)=1-\frac{2 M}{r}+\frac{\beta+q^{2}}{r^{2}}+\frac{1}{240} \frac{\kappa_{5}^{4} q^{4}}{r^{6}} .
$$
tial

The black hole has the associated electromagnetic poten-

$$
A_{\mu} d x^{\mu}=\frac{q}{r} d t
$$

with $q$ being the electric charge. The parameter $\beta$ is interpreted as a tidal charge associated with the bulk Weyl tensor (which may have positive as well as negative values), although $\beta$ can also be considered as a five-dimensional mass parameter $[16,17]$.

\section{The Newman-Janis Algorithm}

In order to obtain the braneworld rotating black hole, we will apply Newman and Janis algorithm [11, 12] starting from the line element (5). Using the outgoing Eddington-Finkelstein coordinates $(u, r, \theta, \varphi)$ we have

$$
d s^{2}=f(r) d u^{2}+2 d u d r-r^{2} d \Omega^{2} .
$$

This metric can be written in terms of null tetrad vectors as

$$
g^{\mu \nu}=l^{\mu} n^{\nu}+l^{\nu} n^{\mu}-m^{\mu} \bar{m}^{\nu}-m^{\nu} \bar{m}^{\mu}
$$

where

$$
\begin{gathered}
l^{\mu}=\delta_{1}^{\mu}, \\
n^{\mu}=\delta_{0}^{\mu}-\frac{1}{2} f(r) \delta_{1}^{\mu}, \\
m^{\mu}=\frac{1}{\sqrt{2} r}\left(\delta_{2}^{\mu}+\frac{i}{\sin \theta} \delta_{3}^{\mu}\right)
\end{gathered}
$$

and $\bar{m}$ denotes the complex conjugate of $m$. These null tetrad vectors satisfy the conditions $l^{\mu} l_{\mu}=n^{\mu} n_{\mu}=m^{\mu} m_{\mu}=l^{\mu} m_{\mu}=$ $n^{\mu} m_{\mu}=0$ and $l^{\mu} n_{\mu}=-m^{\mu} \bar{m}_{\mu}=1$.

Now, we will look at the complexification of the coordinate $r$. As is stressed by Drake and Szekeres [13], there is certain arbitrariness in this process since each term in function $f(r)$ may have its own method of complexification. The mass term depends on $1 / r$, and by following the procedure in [13], it is complexified as

$$
\frac{1}{r} \longrightarrow \frac{1}{2}\left(\frac{1}{r}+\frac{1}{\bar{r}}\right)
$$

On the other hand, the complexification of the terms depending on $\beta$ and $q$ is the key point. We will write these terms as

$$
\frac{\beta+q^{2}}{r^{2}}+\frac{1}{240} \frac{\kappa_{5}^{4} q^{4}}{r^{6}}=\frac{\mathrm{Q}(r)}{r^{2}},
$$

where we defined

$$
Q(r)=\beta+q^{2}+\frac{1}{240} \frac{\kappa_{5}^{4} q^{4}}{r^{4}} .
$$

As shown by Whisker [10], the conventional procedure of the Newman-Janis algorithm does not give a correct solution representing a rotating black hole. The new term $Q(r) / r^{2}$ is similar to the electric charge term in conventional ReissnerNordström solution, and hence, we will follow the proposal of Modesto and Nicolini [14] about the complexification of line elements not comprised by the Newman-Janis algorithm. Suppose that we start from a case in which $Q(r)=Q=$ constant. Since the charge function is a constant, there is no difficulty in following the procedure in [13]; that is, it is unaffected by the complexification and only the transformation

$$
\frac{1}{r^{2}} \longrightarrow \frac{1}{r \bar{r}}
$$

is performed by the algorithm. Let us propose the complexification of our charge term as

$$
\frac{Q(r)}{r^{2}} \longrightarrow \frac{Q[\operatorname{Re}(r)]}{r \bar{r}} .
$$

Then, in order to obtain the correct rotating solution, the function $f(r)$ must transform as

$$
f(r, \bar{r})=1-M\left(\frac{1}{r}+\frac{1}{\bar{r}}\right)+\frac{Q[\operatorname{Re}(r)]}{r \bar{r}},
$$


and hence, the tetrad vectors are

$$
\begin{gathered}
l^{\mu}=\delta_{1}^{\mu}, \\
n^{\mu}=\delta_{0}^{\mu}-\frac{1}{2} f(r, \bar{r}) \delta_{1}^{\mu}, \\
m^{\mu}=\frac{1}{\sqrt{2} \bar{r}}\left(\delta_{2}^{\mu}+\frac{i}{\sin \theta} \delta_{3}^{\mu}\right) .
\end{gathered}
$$

The next step in the algorithm is to perform the complex increments

$$
\begin{aligned}
& r \longrightarrow r^{\prime}=r+i a \cos \theta, \\
& u \longrightarrow u^{\prime}=u-i a \cos \theta
\end{aligned}
$$

on the null tetrad. The function $f(r, \bar{r})$ becomes

$$
f\left(r^{\prime}, \theta\right)=1-\frac{2 M r^{\prime}}{r^{\prime 2}+a^{2} \cos ^{2} \theta}+\frac{Q\left(r^{\prime}\right)}{r^{\prime 2}+a^{2} \cos ^{2} \theta},
$$

and the new tetrad vectors are

$$
\begin{gathered}
l^{\mu}=\delta_{1}^{\mu}, \\
n^{\mu}=\delta_{0}^{\mu}-\frac{1}{2} f\left(r^{\prime}, \theta\right) \delta_{1}^{\mu}, \\
m^{\mu}=\frac{1}{\sqrt{2}\left(r^{\prime}+i a \cos \theta\right)}\left(i a \sin \theta\left(\delta_{0}^{\mu}-\delta_{1}^{\mu}\right)+\delta_{2}^{\mu}+\frac{i}{\sin \theta} \delta_{3}^{\mu}\right) .
\end{gathered}
$$

In order to show that the modification proposal is justified, we need to remember that it was shown in [19] that Schwarzschild-like class of metrics can be cast in the KerrSchild form

$$
d s^{2}=d s_{M}^{2}-\frac{f(r)}{r^{2}}\left(k_{\mu} d x^{\mu}\right)^{2}
$$

where $d s_{M}^{2}$ stands for Minkowski's line element and $k_{\mu}$ is a light-like vector (in the Minkowski coordinates). For Schwarzschild's solution, we simply have $f(r)=2 M r$, while the Reissner-Nordström metric has $f(r)=2 M r-Q^{2}$. In general, we may write $f(r)=2 r M(r)-Q(r)$, as in our case. Now, this kind of decomposition permits to express spinning solutions too. Indeed, even for axisymmetric geometries, we can write

$$
\begin{aligned}
d s^{2} & =d s_{M}^{2}-\frac{f(r)}{r^{2}+a^{2} \cos ^{2} \theta}\left(k_{\mu} d x^{\mu}\right)^{2} \\
& =d s_{M}^{2}-\frac{f(r)}{r \bar{r}}\left(k_{\mu} d x^{\mu}\right)^{2},
\end{aligned}
$$

where the only change is that $k_{\mu}$ is expressed in spheroidal coordinates. As a result of this analysis, we conclude that in all generality the function $f(r)$ is unaffected by the complexification and gives us the justification to propose the complexification of our charge term as given in (16).
Finally, we can read the new metric using (9). Dropping the primes, the line element in the Boyer-Lindquist coordinates is

$$
\begin{aligned}
d s^{2}= & f(r, \theta) d t^{2}-\frac{\Sigma d r^{2}}{a^{2} \sin ^{2} \theta+f(r, \theta) \Sigma} \\
& +2[1-f(r, \theta)] \sin ^{2} \theta d t d \varphi-\Sigma d \theta^{2} \\
& -\sin ^{2} \theta\left[a^{2}(2-f(r, \theta)) \sin ^{2} \theta+\Sigma\right] d \varphi^{2},
\end{aligned}
$$

where

$$
\Sigma=r^{2}+a^{2} \cos ^{2} \theta .
$$

Introducing the quantity

$$
\Delta=r^{2}-2 M r+a^{2}+Q(r),
$$

we express the metric in a more familiar form as follows:

$$
\begin{aligned}
d s^{2}= & \frac{\Delta-a^{2} \sin ^{2} \theta}{\Sigma} d t^{2}-\frac{\Sigma}{\Delta} d r^{2}-\Sigma d \theta^{2} \\
& +2 a \sin ^{2} \theta\left(1-\frac{\Delta-a^{2} \sin ^{2} \theta}{\Sigma}\right) d t d \varphi \\
& -\sin ^{2} \theta\left[\Sigma+a^{2} \sin ^{2} \theta\left(2-\frac{\Delta-a^{2} \sin ^{2} \theta}{\Sigma}\right)\right] d \varphi^{2} .
\end{aligned}
$$

Note that this solution obviously reduces to the static metric (5) when there is no rotation; that is, $a=0$. It is well known that black holes with electric charge are hardly of any astrophysical interest, and thus it is also important to note that the noncharged case, $q=0$, coincides with the tidal KerrNewman black hole in the braneworld first derived in $[20,21]$ and presented also in [10]. Taking in addition $\beta=0$, our solution reduces to conventional Kerr metric, as expected.

Since it is not clear how and why the Newman-Janis algorithm works, it is necessary to explicitly check that the metric (26) indeed solves the Einstein-Maxwell equations on the brane, which we will consider in the contracted form of (1):

$$
R=-8 \pi G T-\frac{\kappa_{5}^{4}}{4}\left(T_{\alpha \beta} T^{\alpha \beta}-\frac{1}{3} T^{2}\right) .
$$

The Ricci scalar calculated from metric (26) is

$$
R=-\frac{\kappa_{5}^{4} q^{4}}{12 r^{6}\left(r^{2}+a^{2} \cos ^{2} \theta\right)} .
$$

This result, together with (27), lets us calculate the associated electromagnetic potential of our solution as

$$
A_{\mu} d x^{\mu}=\frac{q r}{\Sigma}\left(d t-a \sin ^{2} \theta d \phi\right) .
$$

It is important to note that in this paper we did not study the effects of the rotating braneworld black hole on the bulk geometry, and, in particular, the nature of the topological horizon structure in the bulk. Indeed, determining the bulk geometry is an extremely difficult task which needs a deeper study of the projected 5-dimensional bulk Weyl tensor, $E_{\mu \nu}$, which is not considered here. 


\section{Horizons and Thermodynamics}

In complete analogy to the Kerr-Newman solution in general relativity, we define the horizons as the surfaces determined by the roots of

$$
\Delta=r^{2}-2 M r+a^{2}+\beta+q^{2}+\frac{1}{240} \frac{\kappa_{5}^{4} q^{4}}{r^{4}}=0 .
$$

This relation cannot be solved in a closed form, but we can analyse the behavior of the horizons by plotting $\Delta$ as a function of $r$ as shown in Figure 1. Due to the equivalent formal structure of the line element with the Kerr-Newman solution, considerations about horizons are similar. Here we obtain the following three possible cases.

(1) For $M>M_{0}$ there are two distinct horizons $r_{ \pm}$.

(2) For $M=M_{0}$ there is one degenerate horizon $r_{0}$ corresponding to the extremal black hole.

(3) For $M<M_{0}$ there is no horizon and therefore no black hole.

The extremal value $M_{0}$ can be obtained from the conditions

$$
\begin{gathered}
\Delta\left(r_{0}\right)=r_{0}^{2}-2 M r_{0}+a^{2}+\beta+q^{2}+\frac{1}{240} \frac{\kappa_{5}^{4} q^{4}}{r_{0}^{4}}=0 \\
\partial_{r} \Delta\left(r_{0}\right)=2 r_{0}-2 M-\frac{4}{240} \frac{\kappa_{5}^{4} q^{4}}{r_{0}^{5}}=0
\end{gathered}
$$

giving

$$
M_{0}=\sqrt{\frac{24}{25}\left(a^{2}+\beta+q^{2}\right)}
$$

and the degenerate horizon localisation at

$$
r_{0}=\frac{5 M_{0}}{6} .
$$

To complete the analysis of the new solution, we will face the problem of the Hawking temperature. From its definition,

$$
T=\frac{\kappa}{2 \pi}
$$

we need the surface gravity $\kappa$ which can be obtained by the relation

$$
\kappa^{2}=-\frac{1}{2} \nabla^{\mu} \chi^{\nu} \nabla_{\mu} \chi_{v}
$$

where $\chi^{\nu}$ is a null Killing vector. The axisymmetric characteristic of our metric leads us to propose

$$
\chi^{\mu}=\xi^{\mu}+\Omega \zeta^{\mu}
$$

with $\xi^{\mu}=\partial_{t}$ and $\zeta^{\mu}=\partial_{\varphi}$ the being Killing vectors associated with time translation and rotational invariance, respectively. The angular velocity can be found by imposing that

$$
\chi^{\mu} \chi_{\mu}=g_{t t}+2 \Omega g_{t \varphi}+\Omega^{2} g_{\varphi \varphi}=0 \text {. }
$$

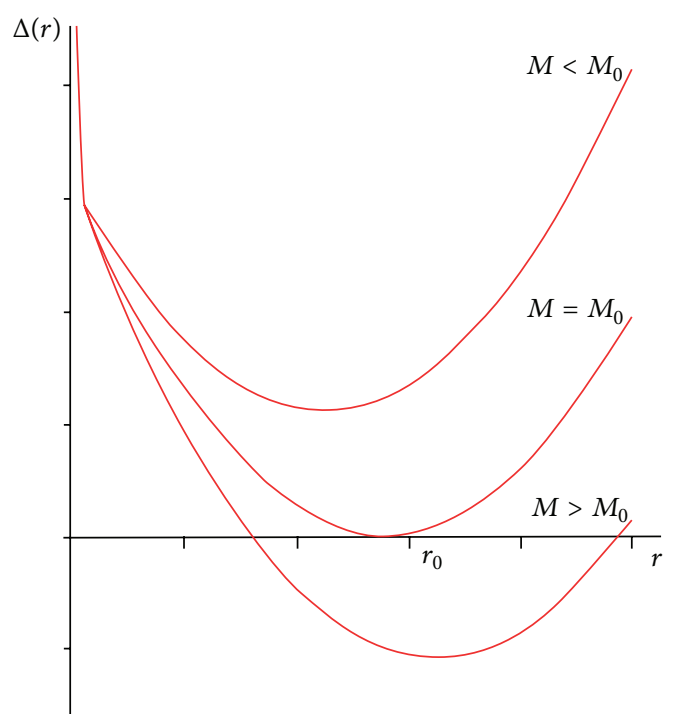

FIGURE 1: Characteristic behaviour of $\Delta(r)$ as a function of $r$ for different values of the mass parameter $M$ using $q=25, \beta=25$, and $a=50$. The intersections with the $r$-axis give the position of inner and outer horizons. For masses $M>M_{0}$, there are two horizons, while the particular case $M=M_{0}$ describes a black hole with one degenerate horizon. For $M<M_{0}$, the behaviour shows no black hole solution

This gives

$$
\begin{gathered}
\Omega=-\frac{g_{t \varphi}}{g_{\varphi \varphi}} \pm \sqrt{\left(\frac{g_{t \varphi}}{g_{\varphi \varphi}}\right)^{2}-\frac{g_{t t}}{g_{\varphi \varphi}}}, \\
\Omega=\omega \pm \frac{\Sigma \sqrt{\Delta}}{\sin \theta\left[\left(a^{2} \sin ^{2} \theta+\Sigma\right)^{2}-\Delta a^{2} \sin ^{2} \theta\right]},
\end{gathered}
$$

where

$$
\omega=-\frac{g_{t \varphi}}{g_{\varphi \varphi}}=\frac{a\left(\Sigma-\Delta+a^{2} \sin ^{2} \theta\right)}{\Sigma^{2}+a^{2} \sin ^{2} \theta\left(2 \Sigma-\Delta+a^{2} \sin ^{2} \theta\right)} .
$$

Note that on the event horizon, $\Delta=0$, the angular velocity reduces to

$$
\Omega_{+}=\omega_{+}=\frac{a}{\left[r_{+}^{2}+a^{2}\right]}
$$

Hence, the surface gravity at the event horizon $r_{+}$can be calculated as

$$
\kappa=\left.\frac{1}{2\left(r_{+}^{2}+a^{2}\right)} \frac{d \Delta}{d r}\right|_{r=r_{+}}
$$

which gives

$$
\kappa=\frac{r_{+}}{2\left(r_{+}^{2}+a^{2}\right)}\left[1-\frac{a^{2}}{r_{+}^{2}}-\frac{\beta+q^{2}}{r_{+}^{2}}-\frac{1}{48} \frac{\kappa_{5}^{4} q^{4}}{r_{+}^{6}}\right] .
$$


This result can be written as

$$
\kappa=\kappa_{\mathrm{KN}}-\frac{r_{+}}{2\left(r_{+}^{2}+a^{2}\right)}\left[\frac{\beta}{r_{+}^{2}}+\frac{1}{48} \frac{\kappa_{5}^{4} q^{4}}{r_{+}^{6}}\right],
$$

where

$$
\kappa_{\mathrm{KN}}=\frac{r_{+}}{2\left(r_{+}^{2}+a^{2}\right)}\left[1-\frac{a^{2}}{r_{+}^{2}}-\frac{q^{2}}{r_{+}^{2}}\right]
$$

is the surface gravity for the conventional Kerr-Newman solution. The temperature associated with our rotating solution is

$$
T=\frac{\kappa}{2 \pi}=T_{\mathrm{KN}}-\frac{r_{+}}{4 \pi\left(r_{+}^{2}+a^{2}\right)}\left[\frac{\beta}{r_{+}^{2}}+\frac{1}{48} \frac{\kappa_{5}^{4} q^{4}}{r_{+}^{6}}\right] .
$$

From the thermodynamical analysis and from Figure 2, it is clear that the possibility of a degenerate horizon gives a temperature that, instead of a divergent behaviour at short scales, admits both a minimum and a maximum before cooling down towards a zero temperature remnant configuration, corresponding to the residual mass $M_{0}$. Note that, for positive tidal charges $(\beta>0)$ the temperature of the black hole is lower than the one corresponding to conventional Kerr-Newman solution $(\beta=0)$. However, for negative tidal charges $(\beta<0)$, our solution has a higher temperature.

\section{Conclusions}

We have obtained a rotating black hole solution in the Randall-Sundrum II braneworld scenario by applying the Newman-Janis algorithm to the topologically charged black hole solution reported by Chamblin et al. $[16,17]$. The new solution carries two types of charge, one arising from the bulk Weyl tensor and one from the gauge field trapped on the brane. In order to obtain this result, we used a modified version of the algorithm in which the involved complexification is the key point. As a future development, we will show that this modified algorithm may work with a wider class of braneworld spacetimes seeds.

The analysis of the horizon structure of the new metric shows similarities to the Kerr-Newmann solution. In particular, there is a minimal mass $M_{o}$ to which the black hole can decay through the Hawking radiation. From the thermodynamical analysis, the possibility of a degenerate horizon gives a temperature that, instead of a divergent behaviour at short scales, admits both a minimum and a maximum before cooling down towards a zero temperature remnant configuration.

As a final remark, we have some comments about future development. In the astrophysical context, charged black holes are often regarded as remote possibilities because, as is well known, a classical analysis shows that the Coulomb repulsion prevents the formation of black holes whose charge/mass ratio exceeds $10^{18}$. On the other hand, the intrinsic quantum gravitational nature of the brane model indicates that these solutions could play an important role in modelling microscopic black hole phenomenology, for example, in the conjectured production at the LHC. Due to the rotation, one might expect intense magnetic fields for the presence of

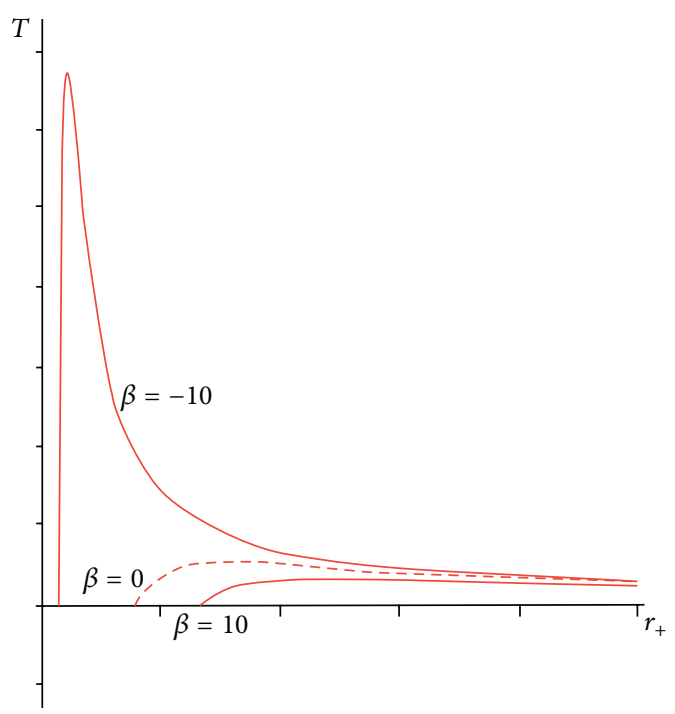

FIGURE 2: Characteristic behaviour of the temperature of the black hole $T$ as a function of the horizon radius $r_{+}$for different values of the parameter $\beta$. In the three plots, we use $q=1$ and $a=1.25$. The dotted line corresponds to the temperature $T_{\mathrm{KN}}$ for the conventional Kerr-Newman solution (i.e., $\beta=0$ ).

superficial currents on the hole. The phenomenology of these magnetic fields would in principle deserve further investigations. Similarly, our new solution can play a role in the context of primordial black holes. It is widely accepted that the early universe could have been populated by tiny black holes, which are nowadays completely evaporated through an scenario which consists of four distinct phases, the spin-down phase (loss of angular momentum), the balding phase (loss of long range fields, i.e., hair, i.e., the electromagnetic charge), the Schwarzschild phase, and the Planck phase. These aspects also deserve further research because in our solution one may expect a new kind of phase in which the black hole cools down to the zero temperature configuration, instead of experiencing the indefinite increase of its temperature. Finally, another research area concerns the sequence of these phases because the discharging time depends on the number of extra dimensions and on the nature of the emission (e.g., brane, bulk brane, or bulk) governing both the Hawking and the Schwinger pair production mechanisms. Hence, our new rotating charged solution provides a first model to study these unexplored aspects.

\section{Acknowledgment}

This work was supported by the Universidad Nacional de Colombia (Hermes Project code 17318).

\section{References}

[1] L. Randall and R. Sundrum, "An alternative to compactification," Physical Review Letters, vol. 83, no. 23, pp. 4690-4693, 1999.

[2] A. Chamblin, S. W. Hawking, and H. S. Reall, "Brane-world black holes," Physical Review D, vol. 61, no. 6, Article ID 065007, 6 pages, 2000. 
[3] S. Nojiri, O. Obregon, S. D. Odintsov, and S. Ogushi, "Dilatonic brane-world black holes, gravity localization, and Newton's constant," Physical Review D, vol. 62, no. 6, Article ID 064017, 10 pages, 2000.

[4] P. Kanti and K. Tamvakis, "Quest for localized 4D black holes in brane worlds," Physical Review D, vol. 65, no. 8, Article ID 084010, 12 pages, 2002.

[5] R. Casadio, A. Fabbri, and L. Mazzacurati, "New black holes in the brane world?" Physical Review D, vol. 65, no. 8, Article ID 084040, 5 pages, 2002.

[6] C. Germani and R. Maartens, "Stars in the braneworld," Physical Review D, vol. 64, no. 12, Article ID 124010, 6 pages, 2001.

[7] R. Casadio and L. Mazzacurati, "Bulk shape of brane-world black holes," Modern Physics Letters A, vol. 18, no. 9, pp. 651660, 2003.

[8] R. Casadio and B. Harms, "Black hole evaporation and large extra dimensions," Physics Letters B, vol. 487, no. 3-4, pp. 209214, 2000.

[9] R. Casadio, "On brane-world black holes and short scale physics," Annals of Physics, vol. 307, no. 2, pp. 195-208, 2003.

[10] R. Whisker, "Braneworld black holes," http://arxiv.org/abs/0810 .1534 .

[11] E. T. Newman and A. I. Janis, "Note on the Kerr spinningparticle metric," Journal of Mathematical Physics, vol. 6, pp. 915917, 1965.

[12] E. T. Newman, E. Couch, K. Chinnapared, A. Exton, A. Prakash, and R. Torrence, "Metric of a rotating, charged mass," Journal of Mathematical Physics, vol. 6, pp. 918-919, 1965.

[13] S. P. Drake and P. Szekeres, "Uniqueness of the Newman-Janis algorithm in generating the Kerr-Newman metric," General Relativity and Gravitation, vol. 32, no. 3, pp. 445-457, 2000.

[14] L. Modesto and P. Nicolini, "Charged rotating noncommutative black holes," Physical Review D, vol. 82, no. 10, Article ID 104035 , 14 pages, 2010.

[15] H. Kim, "Spinning BTZ black hole versus Kerr black hole: a closer look," Physical Review D, vol. 59, no. 6, Article ID 064002, 7 pages, 1999.

[16] A. Chamblin, H. S. Reall, H. A. Shinkai, and T. Shiromizu, "Charged brane-world black holes," Physical Review D, vol. 63, no. 6, Article ID 064015, 11 pages, 2001.

[17] A. Sheykhi and B. Wang, "On topological charged braneworld black holes," Modern Physics Letters A, vol. 24, no. 31, pp. 25312538, 2009.

[18] T. Shiromizu, K. Maeda, and M. Sasaki, “The Einstein equations on the 3-brane world," Physical Review D, vol. 62, no. 2, Article ID 024012, 6 pages, 2000.

[19] A. Smailagic and E. Spallucci, “'Kerrr' black hole: the lord of the string," Physics Letters B, vol. 688, no. 1, pp. 82-87, 2010.

[20] A. N. Aliev and A. E. Gümrükçüoğlu, "Charged rotating black holes on a 3-brane," Physical Review D, vol. 71, no. 10, Article ID 104027, 14 pages, 2005.

[21] A. Aliev, "On recent developments in theoretical and experimental general relativity, gravitation and relativistic field theories," in Proceedings of the 11th Marcel Grossmann Meeting (MG11), pp. 2830-2832, World Scientific, Berlin, Germany, July 2006. 

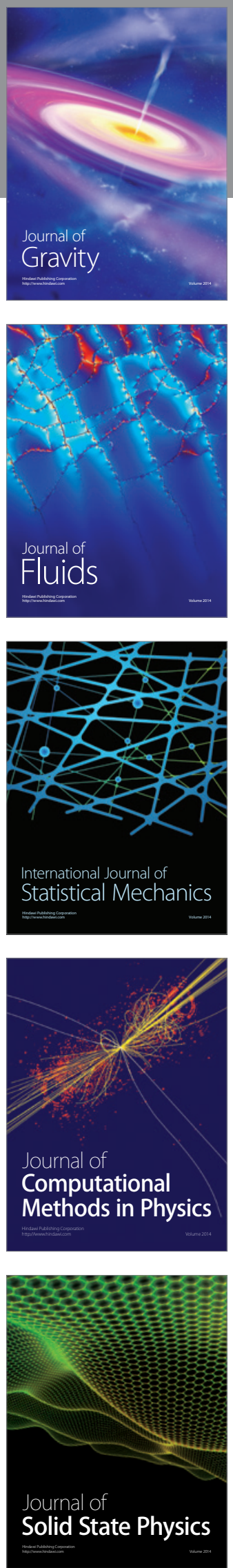

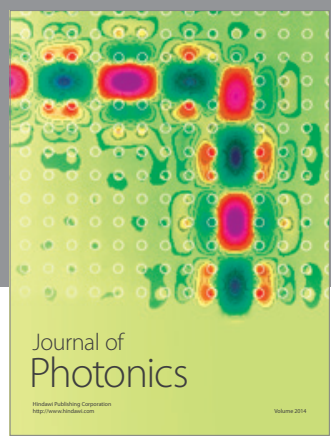

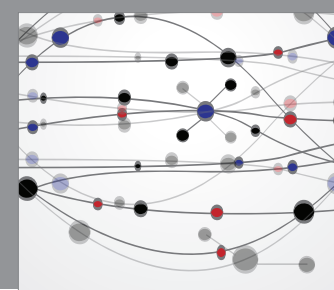

The Scientific World Journal

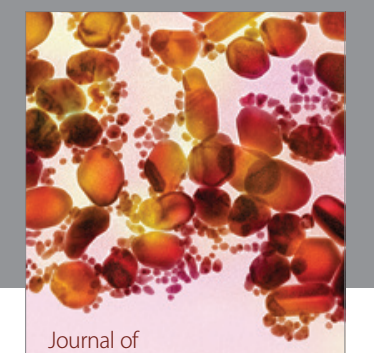

Soft Matter
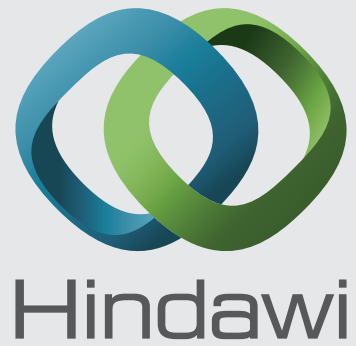

Submit your manuscripts at

http://www.hindawi.com
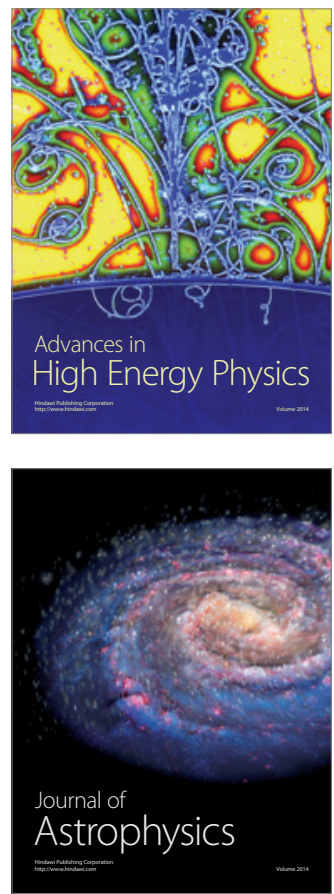
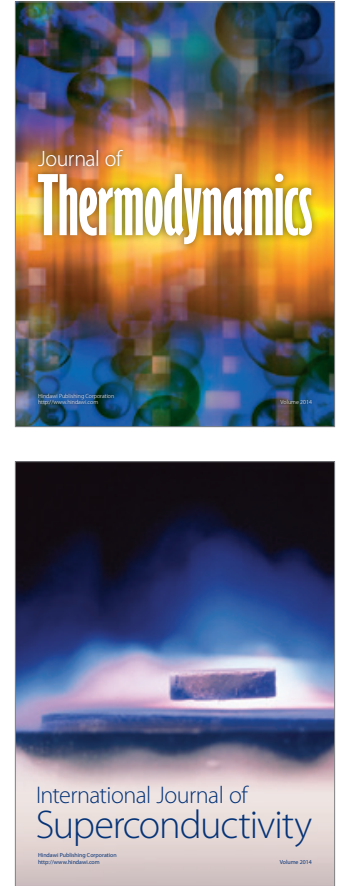
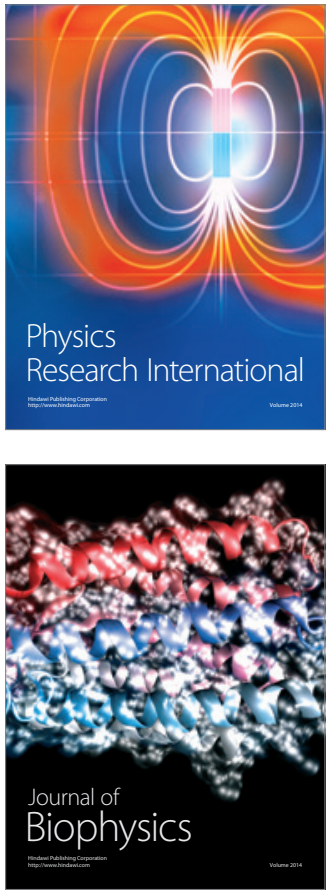
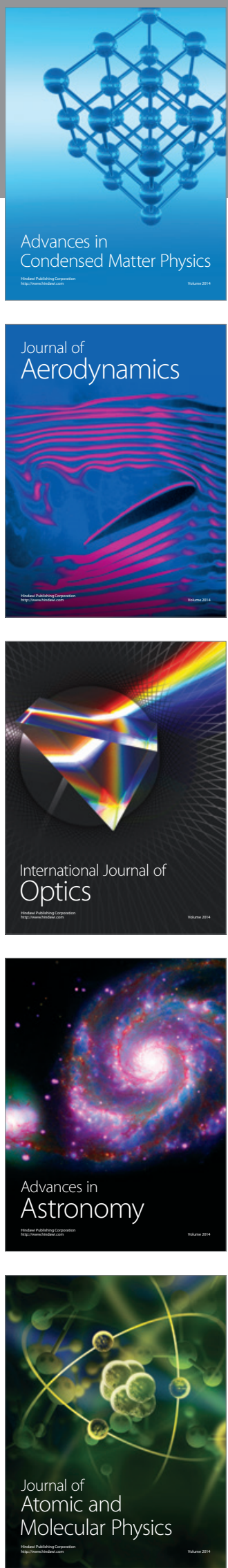